\title{
Principales antecedentes investigativos sobre silencio organizacional
}

\author{
Lic. Evelyn De la llana Pérez \\ Instituto Tecnológico de Formación \\ evelyn.delallana1@formacion.edu.ec
}

\author{
Lic. Rosa Elena Naula Yungaicela \\ Instituto Tecnológico de Formación \\ elena.naula@formacion.edu.ec
}

Recibido: 14 de junio de 2017

Aceptado: 28 de julio de 2017
Cpa. Edith Vanessa Bonin Campos

Instituto Tecnológico de Formación

edith.bonin@formacion.edu.ec

Palabras claves: administración, organizaciones, silencio organizacional.

Keywords: administration, organizations, organizational silence.

\section{RESUMEN}

El silencio en el ámbito laboral tiene importantes consecuencias en el proceso de toma de decisiones pues el mismo puede interferir con la solución de problemas que pueden afectar los niveles de eficiencia y productividad en las empresas. En el presente artículo se pretende analizar las perspectivas de diferentes autores que han abordado la temática del silencio organizacional para dilucidar las diferentes variables e indicadores que permitirán realizar estudios sobre los factores que condicionan el mismo.

\begin{abstract}
Silence in the workplace has important consequences in the decision-making process because it can interfere with the solution of problems that can affect levels of efficiency and productivity in companies. This article aims to analyze the perspectives of different authors who have addressed the issue of organizational silence to elucidate the different variables and indicators that will allow studies on the factors that determine the same.
\end{abstract}




\section{INTRODUCCIÓN}

Las razones que llevan a un profesional a permanecer callado, así como el análisis del significado del silencio y las consecuencias de esta falta de comunicación han sido objeto de estudio de diferentes autores en los últimos años. Sus antecedentes, causas y consecuencias han despertado la curiosidad de los investigadores debido a la importancia que cobra diariamente la comunicación efectiva en las organizaciones.

En un mundo tan cambiante y globalizado, en el que las empresas se hacen cada vez más competitivas, es preciso que cada organización busque la optimización de todos sus procesos y recursos, tanto físicos como humanos, razón por la cual el silencio organizativo podría ser visto como una barrera para el desarrollo de las organizaciones en un mundo pluralista. (Morrison y Milliken, 2000).

En primera instancia el objeto de estudio de Morrison y Milliken (2000) fue la resistencia al cambio por parte de los directivos dadas las creencias implícitas de estos, en las cuales afirman que los empleados son interesados, oportunistas y no están bien informados acerca de la organización, por tal motivo, los directivos tienden a rechazar las retroalimentaciones provenientes de los empleados cuando éstas difieren de su punto de vista.

Debido a lo anterior, surge el interés por estudiar el silencio que guardan los empleados cuando experimentan el rechazo por parte de sus directivos. Diversos académicos se han encargado de estudiar el fenómeno denominado silencio organizativo, y durante varias de estas investigaciones, han surgido muchos otros términos que intentan darle explicación y clasificación. Sin embargo, el silencio abarca múltiples implicaciones, lo que hace que su definición o medición sea compleja.

Hasta el momento no se ha podido descifrar una herramienta que pueda medir con precisión el silencio dentro de las organizaciones, ya que existen muchas variables que no son fácilmente identificables para quienes desean realizar el estudio, por esta razón, los académicos no han podido concluir de manera determinante una forma para medirlo. El presente artículo ofrece una visión general sobre las investigaciones realizadas alrededor del silencio organizativo.

\section{DESARROLLO}

\section{Clasificaciones de Silencio Organizacional}

El silencio organizativo, es definido por Morrison y Milliken, (2000) y Pinder y Harlos (2001) como la decisión consciente de los empleados de reservarse sus opiniones sobre asuntos que conciernen a la organización. Morrison y Milliken (2000) a través del estudio de la estructura organizacional en combinación con las políticas de la empresa concluyeron que el concepto de silencio organizativo varía dependiendo de las dinámicas internas manejadas por la organización.

Por su parte, Pinder y Harlos (2001) investigaron las respuestas y reacciones al silencio organizativo mediante una relación de términos recolectados en investiga- 
ciones previas para establecer un modelo que explicara las etapas donde se evalúa la posición del empleado en cuanto a justicia, los factores que afectan la decisión y las acciones que toma el empleado cuando se siente en una situación de injusticia.

Noëlle-Neumann (1995) plantea que los empleados evalúan el entorno buscando la opinión dominante y expresan su opinión si se asemeja a ésta. Lo que Neumann denomina la espiral del silencio puede manifestarse de forma que la represión de la comunicación va a abstener a los empleados de expresar sus opiniones y en tal sentido plantea:

El resultado es un proceso en espiral que incita a otros individuos a percibir los cambios de opinión y a seguirlos hasta que una opinión se establece como la actitud prevaleciente, mientras que la otra opinión la aportarán y rechazarán todos, a excepción de los duros de espíritu, que todavía persisten en esa opinión. He propuesto el término espiral del silencio para describir este mecanismo psicológico. (p.144).

Para comprender cómo funciona el silencio organizativo dentro de las empresas, es necesario comprender que cada una de ellas es diferente, lo mismo sucede con sus prácticas y dinámicas. Inclusive, los factores que producen o refuerzan el silencio pueden ser imperceptibles para la alta administración. Pinder y Harlos (2001) determinan que hay dos formas de entender el silencio.

La primera la definen como el silencio acústico, el cual hace referencia a la ausencia de sonido, razón que para muchos académicos no es posible, debido a que siempre hay un factor que genera sonido. La segunda forma es el silencio pragmático, en el cual el discurso es ausente; sin embargo, las personas están en constante comunicación lo que significa que a pesar de que se esté en presencia del silencio acústico, se presenta un monólogo interno y constante.

Dentro del silencio pragmático desarrollado por Pinder y Harlos (2001) se puede encontrar otros tipos de silencio que se usa en medio de los discursos. Está el silencio interactivo, el cual consiste en pausas durante las conversaciones, normalmente cargadas de afectos y juicios. El uso indiscriminado del silencio interactivo puede ser interpretado como amenazante y prejuicioso. Éste, por otra parte, permite la reflexión y una forma más consciente de tomar decisiones. Este tipo de silencio es útil para tomar el tiempo para juzgar y pensar sobre las proposiciones de la contraparte.

Otro tipo de silencio que se usa en el discurso es el silencio socio cultural (pro social) que se refieren a los silencios a nivel organizacional y altamente formalizados. Funciona como un mecanismo de control reforzado con autoridad.Son promocionados por normas emitidas dentro de la organización para exigir comportamientos y el mismo puede variar según la cultura, el país o la situación.

Knoll y Van Dick (2011) añadieron otro tipo de silencio a los mostrados por Pinder y Harlos (2001), el silencio oportunista, donde propone que los empleados en ocasiones utilizan la retención de la información para el beneficio propio. 
Para definir este tipo de silencio se basó en la definición de oportunismo como "el interés propio buscado con engaño". (Williamson ,1985).

Van Dyne, Ang y Botero (2003) en sus estudios buscan presentar un marcoconceptual sobre el silencio y la voz como constructos multidimensionales por separado. "La clave para diferenciar estos factores no yace en la presencia o ausencia del sonido, sino en las motivaciones del empleado que retiene sus ideas" (Van Dyne, Ang y Botero, 2003.

Van Dyne, Ang \& Botero (2003) proponen tres tipos de silencio, y a cada uno de ellos le asignan una clasificación de voz para romper cada silencio. El primero de ellos es el silencio de aquiescente, que es la retención de ideas, información y opiniones relevantes basada en la resignación debido al desacople emocional de la organización. Los empleados que enfrentan este tipo de silencio se encuentran en un estado de resignación donde ya no están dispuestos a hablar, envolverse o intentar cambiar la situación por la creencia de que sus aportes no harán la diferencia.

El silencio defensivo se define como la retención de ideas o información basada en el miedo de los empleados a las consecuencias de la expresión de sus criterios; este tipo de silencio funciona de manera defensiva ante amenazas externas. En dicho silencio, los empleados son más proactivos al momento de evaluar posibilidades y escogiendo el silencio como mecanismo de defensa. Por último, se encuentra el silencio pro social el cual se define como la retención de ideas o información con el fin de beneficiar a otras personas de la organización basándose en motivos como la cooperación. (Van Dyne, Ang y Botero 2003).

Este silencio es proactivo e intencional ya que se enfoca en los otros empleados. Al igual que el silencio defensivo, el silencio pro social se fundamenta en la consideración de alternativas sobre la información que se va a retener, pero, por otra parte, el silencio pro social es motivado por la preocupación hacia los otros más que por temor a las consecuencias de usar la voz.

\section{Causas del Silencio Organizacional}

Para una mejor comprensión del silencio organizativo es necesario analizar las causas que dan origen a este fenómeno. Son varias las identificadas por los autores y así mismo, son múltiples los factores que han sido denominados como influyentes en la creación del silencio organizativo.

Morrison y Milliken (2000) definieron en sus estudios dos factores principales que influyen en la creación del silencio organizativo. El primer factor es "miedo de los gerentes a recibir una retroalimentación negativa, un miedo común entre los seres humanos el cual hace que estos perciban las críticas como una amenaza" (p. 708). El segundo factor es el de las creencias implícitas de la gerencia, las cuales son: el creer que los empleados son oportunistas, interesados y que no están bien informados acerca de la organización. "Estas creencias hacen que los gerentes sientan que los empleados no tienen un interés por el bienestar de la organización y que se 
preocupan más por sus intereses personales que por los intereses colectivos de la organización". (Morrison y Milliken, 2000, p. 713).

Pinder y Harlos (2001) agregaron tres factores que definieron como causantes del silencio organizativo. El primer factor es la "Cultura de injusticia", el cual se desarrolla con el fin de explicar la repercusión que tiene para los empleados trabajar en un ambiente laboral injusto, entre esas repercusiones se encuentra el silencio.

El segundo factor es el "Clima del silencio" como las percepciones que se comparten los empleados acerca de lo inútil y peligroso que resulta hablar el cual evita que los implicados den a conocer su desacuerdo u opinión sobre algún aspecto concerniente a la organización, dado que consideran que hacerlo no vale la pena y que adicionalmente puede ser peligroso.

El tercer factor enunciado por Pinder y Harlos (2001) es el "Síndrome del oído sordo", el cual se produce cuando quien recibe una queja u observación por parte del afectado no hace nada al respecto o simplemente hace caso omiso a lo expresado, lo cual causa un gran desánimo y por ende la productividad del mismo se ve afectada.

Vakola y Bouradas (2005) sumaron otras causas a las cuales puede atribuirse en cierta medida la aparición del silencio organizativo. Plantearon que; la falta de apertura a la retroalimentación por parte de la alta gerencia y de los supervisores, está relacionada positivamente con una actitud de silencio por parte de los empleados.

A su vez, las oportunidades de comunicación que se les brindan a los empleados juegan un papel importante, pues estas se relacionan con la aparición de silencio, en tal caso si el empleado percibe que la empresa tiene mecanismos para expresarse y que adicionalmente, estos son eficientes y efectivos, sus actitudes de silencio serán mucho menores, lo contrario a esto acontece cuando los empleados perciben que los mecanismos de comunicación que la organización les brinda son escasos o deficientes .(Vakola y Bouradas, 2005).

Reafirmando la idea sobre el tipo de liderazgo como una de las causas que pueden ser atribuidas al silencio organizativo, se encontró lo planteado por Edmonson (2003) acerca de la incidencia del liderazgo en el silencio dentro de los equipos de traba- jo, especialmente en aquellos que están conformados por grupos interdisciplinarios. Dado que los equipos de acción interdisciplinarios son equipos cuyos miembros poseen habilidades especializadas que deben coordinar entre sí para alcanzar las metas propuestas, la comunicación se convierte en la herramienta más importante para lograr el óptimo funcionamiento del equipo.

Por esta razón Vakola y Bouradas (2005) propusieron que el acompañamiento del líder del equipo facilita el uso de la voz y promueve la expansión de límites en los equipos de acción interdisciplinarios. Detert y Burris (2007) se suman a este grupo cuando afirman que el tipo de liderazgo que los directivos ejecutan es un factor determinante del silencio organizativo, debido a que las características del mismo influyen en gran medida en las actitudes del silencio del empleado, por ejemplo, si el lideraz- 
go está orientado al cambio y a la apertura, los empleados serán más propensos a hablar sobre temas relacionados a la organización y especialmente usaran su voz para buscar la mejora de alguna situación.

Desde otro punto de vista Huang, Van de Vliert y Der Vegt (2005), muestran que las causas del silencio organizativo no sólo deben ser atribuidas a la gerencia o a los supervisores de las organizaciones, pues se encuentra como causantes del silencio mismo a factores que existen independientemente del tipo de liderazgo.

Tal es el caso de las características culturales, pues en su estudio afirman que este factor tiene gran incidencia en el tipo de cultura que se genera dentro de la organización. Adicionalmente discuten que la cultura del país puede influir en la distancia de poder que se maneja de empleados a supervisores, es decir la distancia jerárquica entre gerentes y empleados. Por ejemplo, su investigación demostró que los países con una cultura de gran distancia de poder son menos propensos a expresar sus preocupaciones a colegas o a directivos, pues se les enseña a evitar el conflicto directo con otras personas, a recibir de manera acrítica y obedecer las órdenes de sus jefes.

\section{Factores que influyen en el Silencio Organizacional}

Existen factores individuales, sociales y organizacionales que influyen en la aparición del silencio organizativo los cuales deben ser tomados en cuenta a la hora de realizar estudios de este tipo.

Los factores individuales hacen referencia a los comportamientos individuales de cada miembro de la organización, así como a sus motivaciones y percepciones Para esto, Henriksen y Dayton (2006) definieron tres estados; el primero denominado como la "Disponibilidad Heurística", en el cual se expone la capacidad que tienen los empleados de comportarse según sus códigos o "reglas de oro" y adicionalmente afirman que la mayoría de los errores se producen sistemática y predeciblemente, es decir de manera consciente, por lo cual se resalta la importancia de evaluar la habilidad heurística del personal.

El segundo estado es el "Sesgo por Interés Personal", el cual aparece cuando los empleados solicitan con mayor interés la responsabilidad por los éxitos que los por fracasos propios, razón por la cual optan por darle atribuciones disposicionales a sus éxitos y atribuciones situacionales a sus fracasos. Gracias a su investigación, Henriksen y Dayton (2006) lograron identificar que la mayoría de los empleados tiendena calificar su desempeño por encima de la media, es decir, de manera sobresaliente, cuando esto sucede es muy poca la motivación de los empleados para discutir temas relacionados a la organización y a trabajar en pro de la mejora.

El tercer estado personal identificado por estos estudiosos es La Trampa del Status Quo, el cual hace referencia a una serie de factores que dan lugar a un equilibrio y a un estado de comodidad dentro de la organización, por esto, los empleados no encuentran una motivación de peso para romper dicho estado y tomar un curso de acción diferente. Dado que cambiar el estado de equilibrio dentro de una organiza- 
ción implica toma de decisiones, incertidumbre, duda y renovación de las responsabilidades, resulta fácil para los empleados encontrar razones para no hacer nada y permanecer en silencio, por lo cual, los empleados prefieren no correr riesgos debido a que tienen mayor recordación las fallas producidas por una acción llevada a cabo que por una que no.

Los factores sociales hacen referencia al comportamiento al interior del grupo, el cual brinda una mejor comprensión de los factores que propician el silencio organi- zativo. De estos factores se definieron tres aspectos considerados por los autores como más relevantes. El primero de ellos es la conformidad, que hace referencia a la adaptación de los juicios y creencias propias a las de las demás personas, con el propósito de lograr la aceptación ya sea de un individuo o de un grupo.

El segundo aspecto es la difusión de la responsabilidad que consiste en la tendencia de las personas a asumir menos responsabilidades cuando se trabaja en pro de un objetivo común, entre los miembros del grupo se evitan hablar o discutir sobre los temas relacionados tratando de realizar el mínimo esfuerzo. En tercer lugar, se encuentran los microclimas de desconfianza, los cuales hacen referencia al ambiente que se maneja dentro de la organización gracias a sus supuestos o creencias compartidas.

Para Henriksen y Dayton (2006) estos supuestos o creencias hacen que la percepción que tienen los empleados sobre su clima de trabajo sea prácticamente aceptada, y si el clima de la misma se sustenta en la desconfianza por parte de los miembros de la organización, resultara difícil que alguno se motive a expresarse o a dar su opinión al respecto, fomentando de esa forma la creación del silencio organizativo.

Henriksen y Dayton (2006) enunciaron tres factores organizacionales a los cuales se les debe prestar mayor atención. El primer factor son las creencias no cuestionadas, estas hacen referencia a las suposiciones injustificadas, entre ellas está el creer que, con solo contratar un experto altamente calificado y respetado, todos los conflictos van a desaparecer y automáticamente las buenas decisiones surgirán. La cultura influye mucho en este tipo de creencias que se producen dentro de la organización.

Por ejemplo, si la cultura de una organización es aquella que castiga las decisiones que ocasionan resultados desfavorables, los individuos optarán por no entrometerse en los asuntos de la organización, generando de esta forma un clima desilencio.

El segundo factor organizacional habla sobre las cualidades percibidas del buen trabajador, estas hacen referencia a las características que los líderes y gestores de las organizaciones esperan encontrar en sus trabajadores, no obstante, Henriksen y Dayton (2006) afirman que para evitar el silencio dentro de las organizaciones, es importante que dichas cualidades estén asociadas no solo a tomar la iniciativa o a la capacidad de lidiar con los problemas del día a día, sino que también, deben contribuir al aprendizaje y mejora de la compañía. 
El tercer factor organizacional es el descuido de las interdependencias, este resalta la importancia de comprender las dinámicas y procesos que se desarrollan dentro de la organización, para que las soluciones que se presenten a diferentes asuntos sean efectivas y proporcionen aprendizaje tanto para el empleado como para la compañía.

\section{Efectos del Silencio Organizacional}

Morrison y Milliken (2000) identificaron dos grandes aspectos en los cuales el silencio organizativo tiene grandes implicaciones. En primer lugar, resaltan los efectos de este en la toma de decisiones y en los procesos de cambio de las organizaciones. Dado que la presencia de este fenómeno entorpece la retroalimentación entre directivos y trabajadores y también contribuye en alguna medida a la poca efectividad que se pueda producir en la organización.

En segundo lugar, los autores hacen referencia a las implicaciones del silencio organizativo en las cogniciones, actitudes y comportamientos de los empleados, afirmando que este conlleva a la disminución en la motivación, la insatisfacción y el retiro de los empleados. Esto se debe en gran medida a la poca confianza que los gerentes les otorgan a sus empleados, los cuales terminan sintiendo que no son valorados.

Knoll y Van Dick (2003) propusieron en su estudio las implicaciones de cuatro tipo de silencios específicos. Los cuatro tipos de silencio específicos son: conformista, defensivo, pro social y oportunista y sus implicaciones son: clima de silencio, satisfacción laboral, identificación laboral y bienestar. Estas implicaciones tienen incidencia en el funcionamiento de la organización.

En primer lugar, se encuentra el clima de silencio, el cual, aunque se relaciona con los cuatro tipos de silencio lo hace en mayor proporción con el silencio conformista, lo cual muestra como un clima de silencio es provocado principalmente por el miedo y la resignación. También afirmaron que la satisfacción laboral se relaciona con el silencio conformista y el defensivo, por lo que estos se asocian a un estado de resignación y aceptación de una situación más no de la comodidad con la misma.

Para el caso de la identificación laboral, se da una relación negativa con el silencio conformista, esto se debe a que un indicador importante para la calidad de la relación del empleado con la organización es el grado en que los empleados se sienten identificados y parte de la misma, y la relación positiva se da con el silencio pro social, debido a que un alto grado de afiliación por la compañía y por los compañeros de trabajo genera la necesidad de velar por sus intereses.

En cuanto al Bienestar, Knoll \& Van Dick (2003) enunciaron que este se relaciona negativamente con el silencio conformista y defensivo y positivamente con el silencio oportunista, esto se debe a que el bienestar hace referencia a un estado óptimo laboral, lo cual es opuesto a lo que se enuncia en los silencios conformista y defensivo, y el silencio oportunista se encarga de velar por mantener dicho estado por lo tanto se evidencia la relación directa entre dichas variables. 
Relacionado con la tensión, a mayor silencio conformista y silencio defensivo, mayor será el nivel de tensión de los empleados, esto debido a las características de estos tipos de silencio que afectan y disminuyen su motivación, y a mayor silencio pro social mayor será la tensión, pues en este hay un interés constante por mantener una relación óptima y garantizar el bienestar de los demás miembros de la compañía. Finalmente, sobre la Intención de Rotación, su relación es positiva con el silencio conformista y defensivo, pues resulta una salida más fácil y cómoda al estado de inconformidad en el que se encuentren.

Spencer (1986) resaltó en su estudio la relación del silencio organizativo con la intención de rotación, afirmando que estos se relacionan positivamente, debido a que es más factible que un empleado opte por cambiar o salir de su empleo si no identifica mecanismos y herramientas para expresar la inconformidad que experimenta. Las investigaciones realizadas por Park, C. y Keil (2009) arrojaron una fuerte relación entre el silencio organizativo y el fracaso de un proyecto. A su vez, las estructuras y políticas de la organización, las prácticas gerenciales y el grado de disimilitud demográfica pueden conllevar a la generación del silencio organizativo, lo que finalmente desencadena el fracaso de los proyectos que se formulan en la organización.

\section{CONCLUSIONES}

Esta revisión bibliográfica demuestra que el silencio organizacional está relacionado con múltiples factores, siendo el miedo, la desvinculación y la cooperación algunas de las razones que llevan al empleado a permanecer en silencio. Diferentes ciencias como la Psicología, la Sociología y la Antropología han aportado al estudio del silencio organizacional con importantes indicadores de índole individual, grupal y en el interior de las organizaciones.

El silencio organizativo no es un fenómeno con una definición única y con características generalizables, por el contrario, gracias a los estudios realizados encontramos que en las organizaciones pueden desarrollarse diferentes tipos de silencios, cuyos orígenes están ligados a las características propias del entorno en el que se desarrollan las actividades cotidianas de la organización.

\section{REFERENCIAS BIBLIOGRÁFICAS}

Detert, J.R. y Burris, E.R. (2007). Leadership behavior and employee voice: is the door really open? Academy of Management Journal, Vol. 50 No. 4, pp. 869884.

Henriksen, K y Dayton, E. (2006). Silencio organizacional y el trato Escondido. Health Services Research, Vol. 41, No. 4 p2, pp. 1539-1554.

Knoll, M. y Van Dick, R. (2012). Do I Hear the Whistle...? A First Attempt to Measure Four Forms of Employee Silence and Their Correlates. Journal of Business Ethics. Vol. 113, No. 2, pp. 349-362 
Morrison, E. y Milliken, F. (2000). Organizational Silence: A Barrier to Change and Development in a Pluralistic World. The Academy of Management Review. Vol. 25, No. 4, pp. 706-725.

Noëlle-Neumann, E. (1995). La espiral del silencio. Opinión pública: nuestra piel social. Paidós. Barcelona, pág. 144.

Park, C. \&Keil, M. (2009). Organizational Silence and Whistle-Blowingon IT Projects: An Integrative Model. Decision Sciences. Vol. 40, No. 4, pp. 901-918.

Pinder, C. y Harlos, K. P. (2001). Employee silence: quiescence and acquiescence as responses to perceived injustice. Research in Personnel and Human Resources Management. Vol. 20, pp. 331-369.

Spencer, D. (1986). Employee Voice and Employee Retention. Academy of Management Journal. Vol. 28, No. 3, pp. 488-502.

Vakola, M. y Bouradas, D. (2005). Antecedentes y consecuencias del silencio organizacional. Employee Relations, Vol. 27, pp. 441-458.

Van Dyne, L., Ang, S. y Botero, I. (2003). Conceptualizing employee silence and employee voice as multidimensional constructs. Journal of Management Studies, Vol. 40 No. 6, pp. 1359- 1392.

XuHuang, Evert Van de Vliert\& y Gerben Van der Vegt. (2005). Breaking the Si- lence Culture: Stimulation of Participation and Employee Opinion With holding Crossnationally. Management and Organization Review, 1 Vol. 3, p. 459-482. 